\title{
Interactive comment on "Monitoring of glacier albedo from optical remote-sensing data: application to seasonal and annual surface mass balances quantification in the French Alps for the 2000-2015 period” by Lucas Davaze et al.
}

\section{Davaze \\ lucas.davaze@univ-grenoble-alpes.fr}

Received and published: 28 April 2017

For those interested in the supplementary material of this discussion paper, layout issue can occur for users using both Windows and Firefox. The problem is solved by downloading the supplementary material and opening it outside Firefox.

We're sorry for any inconvenience this might have caused. 